\title{
GEOMETRIC TOPOLOGY AND SHAPE THEORY: A SURVEY OF PROBLEMS AND RESULTS
}

BY B. J. BALL

1. Introduction. The theory of shape, introduced by Karol Borsuk [2] in 1968 , has developed extremely rapidly in the intervening years. Much of the recent work (with the notable exception of that of Borsuk and his students and colleagues in Warsaw) has concentrated on the pro-homotopy, categorical aspect of the theory. I think this may well prove ultimately to be the most important part of the theory-indeed, it may already be so-but nevertheless there remain interesting unsolved problems of a more geometric nature, problems which might be accessible through the more primitive techniques of geometric or general topology. The purpose of this paper is to give a brief (and necessarily incomplete) survey of results of this sort, and to call attention to a number of unsolved problems in this area. With one exception, all the problems listed have appeared in print and are identified with appropriate references. It happens that more than half of the problems listed are due to Borsuk, and a quarter of the total can be found either in his book [15] or in the survey article [14]. These two references are sources of many other problems as well.

2. Basic definitions for compact metric spaces. There are two basic approaches to the shape theory of compact metric spaces: the "fundamental sequences" of Borsuk's initial paper [2] and the "ANR-systems" used by Mardešić and Segal [38], [39]. Since fundamental sequences seem conceptually more geometric in nature, they will be used primarily here. It should be pointed out, however, that some of the results mentioned in this paper were obtained only with the aid of the other approach (which surely is a part of geometric topology, too). Moreover, and more importantly, replacing ANRsequences by ANR-systems extends the notion of shape to all compact Hausdorff spaces.

Let $Q$ denote the Hilbert cube, and consider a sequence $\mathbf{f}=\left\{f_{k}\right\}_{k=1}^{\infty}$ of maps (continuous functions) of $Q$ into $Q$. If $X$ and $Y$ are compact subsets of $Q$, the triple (f, $X, Y$ ) will be called a fundamental sequence from $X$ to $Y$ (in $Q$ ) provided that for every neighborhood $V$ of $Y$, there is a neighborhood $U$ of $X$ such that $f_{i}\left|U \simeq f_{j}\right| U$ in $V$ for almost all integers $i, j$. (Note that $X$ and $Y$ are not uniquely determined by the sequence $\mathbf{f}$; indeed, it is evident that if $(\mathbf{f}, X$, $Y$ ) is a fundamental sequence, then for any compacta $X^{\prime}, Y^{\prime}$ in $Q$ with $X^{\prime} \subset X$ and $Y \subset Y^{\prime}$, (f, $\left.X^{\prime}, Y^{\prime}\right)$ is a fundamental sequence. Thus "restricting the domain and enlarging the range" of a fundamental sequence yields another fundamental sequence. A similar assertion holds for continuous functions, of course.)

Let $\mathbf{i}=\left\{i_{k}\right\}_{k=1}^{\infty}$, where $i_{k}=\mathrm{id}_{Q}$ for all $k$. Then for any compact subset $X$

AMS (MOS) subject classifications (1970). Primary 54-02, 54C99; Secondary 55D10, 55D15.

Key words and phrases. Shape theory, geometric topology.

An expanded version of an invited address delivered at the Tallahassee, Florida meeting of the American Mathematical Society on March 4, 1975; received by the editors April 12, 1976.

(c) American Mathematical Society 1976 
of $Q,(\mathbf{i}, X, X)$ is a fundamental sequence; $(\mathbf{i}, X, X)$ is called the identity fundamental sequence on $X$, and is denoted by $\mathbf{i}_{X}$.

if $X$ is homotopically equivalent to $Y$ then $X$ is fundamentally equivalent to $Y$. Moreover, for the class of (compact) ANR's in $Q$, homotopy and fundamental domination coincide, as do homotopy and fundamental equivalence. To a large extent, the aim of geometric shape theory is to discover relations among arbitrary compacta, relative to these extended notions of equivalence and domination, that are analogous to theorems of homotopy theory for ANR's.

Since every compact metric space can be embedded in $Q$ (and [2] the relations of fundamental equivalence and fundamental domination are independent of the embedding), there is no loss in assuming that all compacta considered are subsets of $Q$. It is worth noting, however, that $Q$ can be replaced in the above definitions by any absolute retract for metrizable spaces which contains $X$ and $Y[\mathbf{1 0}]$. Thus, for example, in considering shapes of compact subsets of $E^{2}$, the fundamental sequences may be taken to consist of maps of $E^{2}$ into itself, rather than having to extend these to all of $Q$.

3. Fundamental retracts. Fundamental retractions and fundamental retracts (or neighborhood retracts) are defined in complete analogy with the usual definitions of retractions and retracts, using fundamental sequences instead of maps. Specifically, if $X$ and $A$ are compact subsets of $Q$ with $A \subset X$, then a fundamental sequence $(\mathbf{r}, X, A)$ is called a fundamental retraction of $X$ to $A$ if $r_{k} \mid A=\mathrm{id}_{A}$ for all $k ; A$ is said to be a fundamental retract of $X$ if there is a fundamental retraction of $X$ to $A$, and $A$ is a fundamental neighborhood retract

Two fundamental sequences $(f, X, Y)$ and $(\mathrm{g}, X, Y)$ are said to be homotopic (denoted $(\mathbf{f}, X, Y) \simeq(\mathrm{g}, X, Y)$ ) if for every neighborhood $V$ of $Y$, there is a neighborhood $U$ of $X$ such that $f_{k}\left|U \simeq g_{k}\right| U$ in $V$ for almost all $k$.

If $\mathbf{f}=\left\{f_{k}\right\}_{k=1}^{\infty}$ and $\mathbf{g}=\left\{g_{k}\right\}_{k=1}^{\infty}$ are sequences of maps of $Q$ into $Q$, then the sequence $\left\{g_{k} f_{k}\right\}_{k=1}^{\infty}$ is called the composition of $\mathbf{f}$ with $\mathbf{g}$, and is denoted by gf. It is easy to see that if (f, $X, Y)$ and $(\mathbf{g}, Y, Z)$ are fundamental sequences, then so is (gf, $X, Z)$; this fundamental sequence is called the composition of (f, $X, Y)$ with $(\mathrm{g}, Y, Z)$.

It is easily verified that homotopy of fundamental sequences is an equivalence relation, and that it is compositive; i.e., if $(\mathbf{f}, X, Y) \simeq\left(\mathbf{f}^{\prime}, X, Y\right)$ and $(\mathbf{g}$, $Y, Z) \simeq\left(\mathbf{g}^{\prime}, Y, Z\right)$, then $(\mathbf{g f}, X, Z) \simeq\left(\mathbf{g}^{\prime} \mathbf{f}^{\prime}, X, Z\right)$.

Two compact subsets $X, Y$ of $Q$ are said to be fundamentally equivalent, or to have the same shape, if there exist fundamental sequences $(f, X, Y)$ and $(\mathrm{g}$, $Y, X)$ which are homotopy inverses; i.e., $($ gf, $X, X) \simeq \mathbf{i}_{X}$ and $(\mathbf{f g}, Y, Y) \simeq \mathbf{i}_{Y}$. The class of all compact subsets of $Q$ having the same shape as a given compactum $X$ in $Q$ is denoted by $\operatorname{Sh}(X)$. Thus two compacta $X, Y$ in $Q$ have the same shape if and only if $\operatorname{Sh}(X)=\operatorname{Sh}(Y)$, or, equivalently, if $Y \in \operatorname{Sh} X$.

If only the first of the two homotopy relations above is postulated (i.e., (gf, $X, X) \simeq \mathbf{i}_{X}$ ), then $X$ is said to be fundamentally dominated (or shape dominated) by $Y$; this is expressed notationally by $\operatorname{Sh}(X) \leqslant \operatorname{Sh}(Y)$. A property $\alpha$ of compacta is called a shape invariant (hereditary shape invariant) provided that if $Y$ has property $\alpha$, so does every compactum $X$ which has the same shape as $Y$ (respectively, which is shape dominated by $Y$ ).

The relations of fundamental equivalence and fundamental domination are 
extensions of the concepts of homotopy equivalence and homotopy domination, in the sense that for any two compact subsets $X, Y$ of $Q$, if $X$ is homotopically dominated by $Y$, then $X$ is fundamentally dominated by $Y$ and of $X$ if there exist a closed neighborhood $N$ of $A$ in $X$ and a fundamental retraction of $N$ to $A$. The definitions of fundamental absolute retract (FAR) and fundamental absolute neighborhood retract (FANR) are entirely analogous to the corresponding definitions of AR's and ANR's, using fundamental retractions in place of retractions. Among the basic properties of FAR's and FANR's are the following, all due to Borsuk [3].

(3.1) Every AR in $Q$ is an FAR and every ANR in $Q$ is an FANR.

(3.2) Every fundamental retract of an FAR (FANR) is an FAR (FANR).

(3.3) A compactum in $Q$ is an FAR (FANR) if and only if it is a fundamental retract of an AR (ANR).

(3.4) All Betti groups of an FANR are finitely generated and almost all are trivial. (In particular, an FANR can have only a finite number of components. It easily follows that every component of an FANR is an FANR.)

(3.5) A compactum $X$ in $E^{2}$ is an FANR if and only if $X$ has only a finite number of components, and each component of $X$ has only a finite number of complementary domains in $E^{2}$.

It is also true [9] that a compactum $X$ in $Q$ is an FAR if and only if $X$ has trivial shape (i.e., the shape of a point). Thus $X$ is an FAR if and only if $X$ has the shape of an AR, and it is natural to conjecture that $X$ is an FANR if and only if $X$ has the shape of an ANR, or of a polyhedron [14]. This conjecture has recently been disproved: Edwards and Geoghegan [22] have given an example of a (2-dimensional) continuum $X$ which is an FANR but does not have the shape of any finite complex; moreover, West [54] has shown that every ANR has the homotopy type (and therefore the shape) of a finite complex, so $X$ does not have the shape of any ANR. It follows from (3.5) that every FANR lying in $E^{2}$ does have the shape of a polyhedron, and it is known [51] that every 1-dimensional FANR has the shape of a plane compactum. So the question of which FANR's have polyhedral shape is completely settled with respect to dimension: all 0 - and 1-dimensional ones do, those of dimension 2 or more need not. However, the following questions remain open.

Problem 1 [15, p. 350]. Does every FANR in $E^{3}$ have the shape of a polyhedron?

Problem 2 [15, p. 357]. Does every movable (definition in \$4) compactum in $E^{3}$ with finite Betti numbers have the shape of a polyhedron?

In analogy with well-known results for AR's, Borsuk [3] proved that if $X, Y$ and $X \cap Y$ are FAR's, then $X \cup Y$ is an FAR, and Chapman [18] has shown that if $X \cap Y$ and $X \cup Y$ are FAR's, then $X$ and $Y$ are FAR's. It is natural to inquire whether similar results hold for FANR's.

Problem 3 [3], [13]. If $X, Y$ and $X \cap Y$ are FANR's, must $X \cup Y$ be an FANR?

It is known that the intersection of any decreasing sequence of AR's is an FAR, but it is easy to see that the corresponding result for ANR's is false. Borsuk has shown, however, that if $Y_{1} \supset Y_{2} \supset \ldots$ is a sequence of ANR's such that $Y_{k+1}$ is a deformation retract of $Y_{k}$ for all $k$, then $\cap_{k=1}^{\infty} Y_{k}$ is an FANR. 
Problem 4 [3]. If $Y_{1} \supset Y_{2} \supset \ldots$ is a sequence of ANR's such that $Y_{k+1}$ is a retract of $Y_{k}$ for each $k$, is $\cap_{k=1}^{\infty} Y_{k}$ an FANR?

Problem 5 [3]. If $Y_{1} \supset Y_{2} \supset \ldots$ is a sequence of ANR's and $Y_{k+1}$ is a retract of $Y_{k}$ for each $k$, must $Y_{k+1}$ be a deformation retract of $Y_{k}$ for all sufficiently large $k$ ?

Problem 6 [3]. If $X$ is an FANR and $Y_{1} \supset Y_{2} \supset \ldots$ is a sequence of fundamental retracts of $X$, is $\cap_{k=1}^{\infty} Y_{k}$ a fundamental retract of $X$ ? (It is known [20] that this is false without the hypothesis that $X$ be an FANR.)

4. Movability. The notion of a movable space seems to be one of the most useful ideas in shape theory. The basic definitions are due to Borsuk ([4], [8], [9]).

A compact set $X$ in $Q$ is said to be movable if for every neighborhood $U$ of $X$, there is a neighborhood $U_{0}$ of $X$ which can be deformed into any neighborhood of $X$ by a homotopy in $U$; i.e., for every neighborhood $V$ of $X$, there is a homotopy $\varphi_{V}: U_{0} \times I \rightarrow U$ such that $\varphi_{V}(x, 0)=x$ and $\varphi_{V}(x$, 1) $\in V$ for every $x \in U_{0}$. If the homotopy $\varphi_{V}$ can always be chosen so that $\varphi_{V}(x, 1)=x$ for every $x \in X$, then $X$ is said to be strongly movable. If $x_{0} \in X$, the pair $\left(X, x_{0}\right)$ is pointed movable, or $X$ is movable with respect to $x_{0}$, if $\varphi_{V}$ can be chosen so as to leave $x_{0}$ fixed at all times $t$.

Related to the idea of pointed movability is that of shapes of pointed compacta, or pointed shapes. These are defined and their basic properties demonstrated on pp. 243-246 of [2], and will not be repeated in detail here. Suffice it to say that for a pointed fundamental sequence from $\left(X, x_{0}\right)$ to $\left(Y, y_{0}\right)$ one requires a sequence $\mathbf{f}=\left\{f_{k}\right\}_{k=1}^{\infty}$ of maps of $\left(Q, x_{0}\right)$ into $\left(Q, y_{0}\right)$; moreover, all homotopies in the definition of "pointed fundamental sequence" are required to be rel $x_{0}$, as are the homotopies involved in the definition of homotopic pointed fundamental sequences. (Recall that a map $\varphi: A \times I \rightarrow B$ is a homotopy rel $x_{0}$, where $x_{0} \in A$, if for each $t \in I, \varphi\left(x_{0}\right.$, $t)=\varphi\left(x_{0}, 0\right)$.) The pointed shape, $\operatorname{Sh}\left(X, x_{0}\right)$, of a pointed compactum is defined analogously to $\operatorname{Sh}(X)$, and similarly for the relation $\operatorname{Sh}\left(X, x_{0}\right) \leqslant$ $\operatorname{Sh}\left(Y, y_{0}\right)$. (Perhaps it is well to point out that $\operatorname{Sh}\left(X, x_{0}\right)$ is not the same as $\operatorname{Sh}\left(X,\left\{x_{0}\right\}\right)$, where $\operatorname{Sh}\left(X,\left\{x_{0}\right\}\right)$ is the (relative) shape of the pair $\left(X, X_{0}\right)$, defined earlier in [2], with $X_{0}=\left\{x_{0}\right\}$.)

It has been shown by Borsuk ([4], [8]) that movability (pointed movability) is an hereditary invariant of shape (pointed shape).

Other results on movability include the facts [4] that every ANR, or more generally, every FANR is movable, that every plane compactum is movable (in fact [8], pointed movable with respect to each of its points), that solenoids are not movable, and that a compactum is movable if each of its components is movable (but not conversely). Additionally [8], if $(X, a)$ is movable, so is $(X, b)$ for any point $b$ in the same component of $X$ as $a$; from this hypothesis $((X, a)$ movable and $b$ in the component of $X$ containing $a)$ it follows that $\operatorname{Sh}(X, a)=\operatorname{Sh}(X, b)$. This latter result does not hold in general without the requirement that $(X, a)$ be movable. It was shown in [3] that every FANR is strongly movable (without using that term), and in [9] it is shown that strong movability in fact characterizes FANR's. The question of whether, or when, movability implies pointed movability is of considerable interest; the question 
has been raised independently by Borsuk [8], McMillan [41] and Krasinkiewicz [31]. Though phrased in point set topology terms, this question seems to be essentially algebraic in nature. Nevertheless, because of its importance to many shape theory results, it is included here.

Problem 7 [8], [41], [31]. Is every movable continuum $X$ necessarily pointed movable with respect to one (or to each) of its points?

A related result, given by McMillan [41], is that if $X$ is a movable continuum and $\left(X, x_{0}\right)$ is pointed 1-movable, then $\left(X, x_{0}\right)$ is movable.

Mardešić has shown [34] that every $n$-dimensional $L C^{n-1}$ continuum is movable. Borsuk [5] gave an example of a nonmovable locally connected $\left(L C^{0}\right)$ continuum $X_{0}$ in $E^{3}$, with $\operatorname{dim} X_{0}=2$. The continuum $X_{0}$, indicated in Figure 1, is locally $E^{2}$ at all points except $a$.

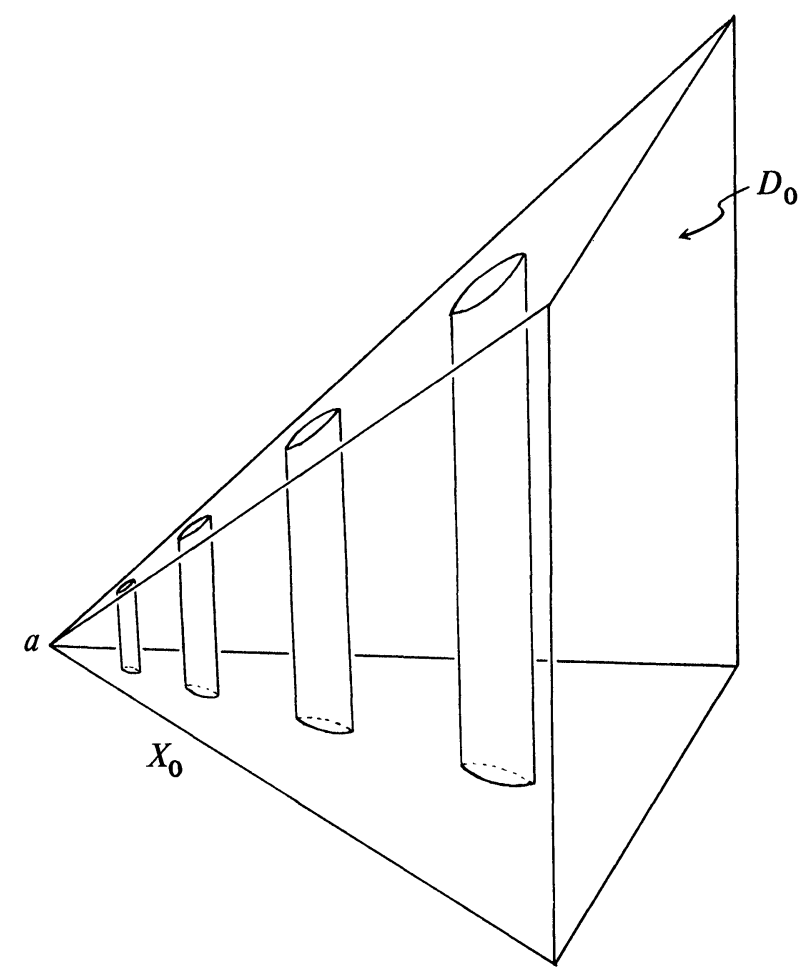

FIGURE 1

Borsuk showed that $X_{0}-$ Int $D_{0}$ has the shape of a plane continuum, and hence is movable.

Problem 8 [5]. Is every closed proper subset of $X_{0}$ movable?

In connection with the preceding example of Borsuk, we note that McMillan [42] has recently constructed a locally connected nonmovable continuum in $E^{3}$ which does not separate $E^{3}$.

Of course, there exist many nonlocally connected continua which are movable (for example in $E^{2}$ ), but there are no known movable continua which do not at least have the shape of a locally connected continuum. This is an example of what Borsuk calls the problem of "reasonable representatives of shapes." 
Problem 9 [15, p. 357]. Does every movable continuum have the shape of a locally connected continuum?

In an interesting recent paper [31], J. Krasinkiewicz has shown, among many other results, that every 1-dimensional continuous image of a tree-like continuum is movable. He poses several questions, of which the following two seem most relevant here.

Problem 10 [31]. Is every 1-dimensional continuous image of a movable continuum movable?

Problem 11 [31]. Are all arcwise connected 1-dimensional continua movable?

A compact subset $X$ of $Q$ is said to be $n$-movable [11] if for every neighborhood $U$ of $X$, there is a neighborhood $U_{0}$ of $X$ such that any $n$-dimensional subset of $U_{0}$ can be deformed into an arbitrary neighborhood of $X$ by a homotopy with values in $U$. Borsuk shows that $n$-movability is an hereditary shape invariant, that a compactum is $n$-movable if each of its components is $n$-movable, and that the suspension of any $n$-movable compactum is $n$-movable. He poses the following question.

Problem 12 [11]. If $X$ is $n$-movable and $Y$ is $m$-movable, must $X \times Y$ be $(n+m)$-movable?

A partial answer to Problem 12 has been given by Kodama and Watanabe [29], who show that, under the given hypothesis, $X \times Y$ is $k$-movable where $k=\min (n, m)$. Kodama and Watanabe also answer another question of Borsuk by showing that there is a nonmovable continuum which is $n$-movable for every $n$; the same example was also given independently by Kozlowski and Segal [30].

McMillan [41] has introduced an interesting variant of 1-movability, which he calls "nearly 1-movable." McMillan indicates that solenoids fail to be nearly 1-movable, as does the Case-Chamberlain curve, and shows how to construct an example of a 1-dimensional continuum which is nearly 1-movable but not 1-movable. He also proves that every continuous image of a nearly 1 -movable continuum is nearly 1 -movable, and that every continuous image of a pointed 1-movable continuum is pointed 1-movable.

PROBLEM 13 [41]. Is every continuous image of a 1-movable continuum necessarily 1 -movable?

The next question, a special case of Problem 6, is also due to McMillan.

PROBLEM 14 [41]. Is every 1 -movable continuum necessarily pointed 1 -movable (with respect to at least one of its points)?

Another result from [41] is that if $A$ and $B$ are nondegenerate continua such that $A \times B$ is embeddable in a PL 3-manifold, then each of $A$ and $B$ is pointed 1-movable with respect to each of its points. This suggests the following question.

Problem 15 [41]. If $A$ and $B$ are nondegenerate continua such that $A \times B$ is embeddable in a PL 3-manifold, must each of $A$ and $B$ be movable?

N. Shrikandhe [49] has shown that if $X$ is a compactum in $E^{n}$, then $E^{n} / X$ is locally simply connected if and only if $X$ is nearly 1-movable. McMillan and Shrikandhe [43] have used this theorem to obtain a number of additional results on the simple connectivity of quotient spaces. 
5. Fundamental dimension and Euclidean coefficients. The Euclidean coefficient, $e(X)$, of a compactum $X$ is the smallest $n(n=0,1, \ldots, \infty)$ such that $\operatorname{Sh}(X) \leqslant \operatorname{Sh}(Y)$ for some compactum $Y \subset I^{n}$. This definition of $e(X)$ is (equivalent to) that given in [15]. In [14], $e(X)$ was defined to be the smallest $n$ such that $\operatorname{Sh}(X)=\operatorname{Sh}(Y)$ for some $Y \subset I^{n}$; here we will let $e^{\prime}(X)$ denote this latter number. It is clear that $e(X) \leqslant e^{\prime}(X)$ and that $\operatorname{Sh}(X) \leqslant \operatorname{Sh}(Y)$ implies $e(X) \leqslant e(Y)$. The following problems remain open, however.

Problem $16\left[15\right.$, p. 354]. Is $e(X)=e^{\prime}(X)$ for every compactum $X$ ?

Problem 17 [14]. Does $\operatorname{Sh}(X) \leqslant \operatorname{Sh}(Y)$ imply $e^{\prime}(X) \leqslant e^{\prime}(Y)$ ?

Several other interesting questions concerning the numbers $e(X)$ and $e^{\prime}(X)$ are given in [14] and [15].

The fundamental dimension $\mathrm{Fd}(X)$ of a compactum $X$ was introduced in [7]; it is the smallest $n(n=0,1, \ldots, \infty)$ such that $\operatorname{Sh}(X) \leqslant \operatorname{Sh}(Y)$ for some $n$-dimensional compactum $Y$. (Equivalently [45], [28], $\mathrm{Fd}(X)$ is the smallest $n$ such that $\operatorname{Sh}(X)=\operatorname{Sh}(Y)$ for some $n$-dimensional $Y$.)

The fundamental dimension has been studied extensively by $\mathrm{S}$. Nowak ([45], [46], [47]). The following are some of his results.

(5.1) For any compactum $X \subset Q, \operatorname{Fd}(X) \leqslant n$ if and only if $X$ is deformable inside each of its neighborhoods to a set of dimension $\leqslant n$.

(5.2) For any compactum $X \subset Q, \operatorname{Fd}(X) \leqslant n$ if and only if $\operatorname{Fd}(C) \leqslant n$ for every component $C$ of $X$.

(5.3) Every compact, connected $n$-manifold with nonempty boundary has fundamental dimension $\leqslant n-1$.

(5.4) Every compact proper subset of a PL $n$-manifold has fundamental dimension $\leqslant n-1$; in particular, $\operatorname{Fd}(X) \leqslant n-1$ for every compact subset of $E^{n}$.

PROBLEM 18 [45]. Does every compact proper subset of an $n$-manifold have fundamental dimension $\leqslant n-1$ ?

Problem 19 [46]. If $\operatorname{Fd}(X)>\operatorname{Fd}(X \cap Y)$, is $\operatorname{Fd}(X \cup Y) \geqslant \operatorname{Fd}(X)$ ?

Problem 20 [46]. Let $C$ be the well-known Case-Chamberlain curve. Is $\operatorname{Fd}\left(C^{n}\right)=n$ ?

The following question is due to Borsuk.

Problem 21 [14], [15]. For every compactum $X$, is $\operatorname{Fd}\left(X \times S^{1}\right)=\operatorname{Fd}(X)+$ 1? More generally, is $\operatorname{Fd}\left(X \times S^{n}\right)=\operatorname{Fd}(X)+n$ ?

Kodama [28] has obtained a number of results on fundamental dimension, and has defined the fundamental dimension $\operatorname{Fd}(X, A)$ for a pair of compacta as the minimum dimension of a compactum $Y$ having a closed subset $B$ such that $\operatorname{Sh}(X, A) \leqslant \operatorname{Sh}(Y, B)$. (Here $\operatorname{Sh}(X, A)$ is the relative shape defined in [2].) Kodama shows that if $X$ is an AR and $A$ is a closed subset of $X$, then $\operatorname{Fd}(A) \leqslant \operatorname{Fd}(X, A) \leqslant \operatorname{Fd}(A)+1$. He asks the following question.

Problem 22 [28]. For every compact pair $(X, A)$, is $\operatorname{Fd}(X, A) \leqslant$ $\max (\operatorname{Fd}(X), \operatorname{Fd}(A)+1) ?$

6. Stability. A space $X$ is said to be homotopically stable (or $H$-stable) if for each closed proper subset $Y$ of $X$, no map of $X$ into $Y$ is homotopic in $X$ to $\mathrm{id}_{X}$. Similarly, $X$ is $R$-stable if no proper subset of $X$ is a deformation retract of $X$. Borsuk [13] has studied analogs of these notions in shape theory and has raised a number of interesting questions concerning them. 
A compact subset $X$ of $Q$ is fundamentally stable, or F-stable, if for no closed proper subset $Y$ of $X$ is there a fundamental sequence (f, $X, Y)$ with (f, $X, X) \simeq \mathbf{i}_{X}$. Similarly, $X$ is fundamentally $R$-stable, or FR-stable, if no closed proper subset of $X$ is a fundamental deformation retract of $X$ (i.e., for no closed proper subset $Y$ of $X$ is there a fundamental retraction $(\mathbf{r}, X, Y)$ with $\left.(\mathbf{r}, X, X) \simeq \mathbf{i}_{X}\right)$. Finally, $X$ is shape stable, or $S$-stable, if no closed proper subset of $X$ has the same shape as $X$. (Shape stable compacta are called "primitive" in [15].) Borsuk obtains a number of theorems related to these notions, including in particular the result that every FANR $X$ contains an $F R$-stable compactum $Y$ with $\operatorname{Sh}(Y)=\operatorname{Sh}(X)$. He poses, among others, the following similar questions.

Problem 23 [13]. Does every compactum $X$ contain an $F R$-stable (or an $F$-stable) compactum $Y$ with $\operatorname{Sh}(Y)=\operatorname{Sh}(X)$ ?

Problem 24 [13]. Does every continuum $X$ contain an $S$-stable continuum $Y$ with $\operatorname{Sh}(Y)=\operatorname{Sh}(X)$ ?

An affirmative answer to Problem 24 for the case $\operatorname{Fd}(X)<\infty$ has been given by Cook, Feuerbacher and Kuperberg [19].

Problem 25 [15, p. 357]. Is the product (or the one-point union) of two $S$-stable compacta necessarily $S$-stable?

7. Shapes and complements. The beautiful result of Chapman [16] that two $Z$-sets in $Q$ have the same shape if and only if their complements in $Q$ are homeomorphic has inspired a number of investigations concerning similar theorems for compacta in $E^{n}$ or $S^{n}$.

First, Chapman [17] proved a finite dimensional version of his theorem, but needed strong codimension requirements as well as a fairly complex embedding condition. Geoghegan and Summerhill [25] reduced the codimension requirements to the trivial range, the best possible for arbitrary compacta, and replaced Chapman's embedding condition with the more familiar 1-ULC complement property. Rushing [48], considering embeddings in $S^{n}, n \geqslant 5$, showed that if one of the compacta is $S^{k}(k \neq 1)$ and the other is globally 1 -alg, no further restriction is needed to obtain the equivalence of "having the same shape" and "having homeomorphic complements" (except for the case $k=n-2$, which can be handled by adding an embedding restriction). Hollingsworth and Rushing [27] show that, for compacta in $E^{n}, n \geqslant 5$, with dimensions in the trivial range, it is sufficient that $X$ and $Y$ satisfy the "small loops condition." Additional results of this kind have been obtained by Liem [32] and Venema [52], [53]. Coram, Daverman and Duvall [20] have obtained results relating shapes and embeddings in $E^{n}$ of a different sort, showing that, under suitable dimensional and embedding restrictions, a compactum in $E^{n}$ which has the shape of a complex $K$ must have arbitrarily close neighborhoods which are regular neighborhoods of a copy of $K$ in $E^{n}$.

It seems likely that further results relating shapes of compacta in $E^{n}$ to properties of their embeddings will be developed, but apparently no specific problems in this area have been posed in the literature.

8. Compact Hausdorff spaces. As indicated in $\$ 2$, the theory of shape can be extended to the class of all compact Hausdorff spaces by the method of Mardešić-Segal, based on inverse systems of ANR's. Since the definitions are 
fairly involved and only a few problems in this area will be mentioned, the definitions will not be repeated here. The interested reader is referred to [38] and [39].

Gordh and Mardešić [26], using categorical techniques, obtain a number of results on movable Hausdorff curves (1-dimensional continua). They ask several questions, including the following.

Problem 26 [26]. Is every locally connected (hereditarily locally connected) Hausdorff curve movable?

Mardešić [33] has generalized the notion of FANR to compact Hausdorff spaces, and uses the terminology absolute neighborhood shape retract (ANSR) for this generalized concept.

Mardešić has also [35] extended the definition of strong movability to compact Hausdorff spaces. He shows, among other results, that every ANSR is strongly movable. Borsuk [9] proved that strong movability (of compact metric spaces) characterizes FANR's, and Mardešić asks whether the analogous result holds in his more general setting.

PROBLEM 27 [35]. Is every strongly movable compact Hausdorff space an ANSR?

The following question was asked by D. A. Edwards in a casual conversation. It probably has not been seriously considered by anyone, but would be interesting if true.

Problem 28. If $X$ is an open $n$-cell and $\beta X$ is the Cech compactification of $X$, is $\operatorname{Sh}(\beta X-X)=\operatorname{Sh}\left(S^{n-1}\right)$ ?

9. Noncompact spaces. There are several ways of extending shape theory to noncompact spaces; there seems to be general agreement that the approach of Mardešić [36] (see also Morita [44]) or an equivalent method is the most appropriate. This treatment applies to arbitrary topological spaces, and is highly abstract and nonintuitive. If one is willing to restrict attention to a smaller class of spaces, however, more geometrically oriented theories are available.

For metrizable spaces, the shape theory developed by Fox ([23], [24]) does not completely obscure the geometry involved (and is equivalent [37] to Mardešić's theory restricted to metrizable spaces).

Borsuk's "weak shape" for metrizable spaces [12], [15] and the related notion of "position" (of a set in a space) are quite geometrically oriented. There are many appealing problems here.

The theory of proper shape for locally compact metric spaces, introduced by the author and R. B. Sher [1], was geometrically motivated. It was an attempt to carry over to a class of noncompact spaces the property of Borsuk's shape theory for compacta of reflecting the global geometric similarities of spaces while ignoring their local complexities. It might be worth further development.

10. Concluding remarks. It has not been possible here to give a truly comprehensive survey of geometrically appealing results and problems of shape theory. Many topics have been treated less thoroughly than they deserve, and many others have been omitted altogether. However, in addition to the references cited explicitly, I have attached a supplementary bibliogra- 
phy listing many other papers that seem to fall into the category of "geometric shape theory." I am sure there are omissions in this listing, but I hope it will prove useful to those who wish to undertake a systematic study of the area. In this connection, I should remark that Jack Segal [55] has compiled an essentially complete bibliography of shape theory.

\section{REFERENCES}

1. B. J. Ball and R. B. Sher, $A$ theory of proper shape for locally compact metric spaces, Fund. Math. 86 (1974), 163-192. MR 51 \# 1724.

2. K. Borsuk, Concerning homotopy properties of compacta, Fund. Math. 62 (1968), 223-254. MR 37 \#4811.

3. _ Fundamental retracts and extensions of fundamental sequences, Fund. Math. 64 (1969), 55-85; errata, ibid. 64 (1969), 375. MR 39 \#4841.

4. - On movable compacta, Fund. Math. 66 (1969/70), 137-146. MR 40 \# 4925.

5. _ On a locally connected non-movable continuum, Bull. Acad. Polon. Sci. Sér. Sci. Math. Astronom. Phys. 17 (1969), 425-430. MR 41 \# 1012.

6. , On the shape of FANR-sets, Bull. Acad. Polon. Sci. Sér. Sci. Math. Astronom. Phys. 17 (1969), 529-532. MR 41 \# 1050.

7. Concerning the notion of the shape of compacta, Proc. Internat. Sympos. on Topology and its Applications (Herceg-Novi, 1968), Savez Društava Mat. Fiz. i Astronom., Belgrade, 1969, pp. 98-104. MR 43 \# 1138.

8. Some remarks concerning the shape of pointed compacta, Fund. Math. 67 (1970), 221-240. MR 42 \#1076.

9. $\quad$ A note on the theory of shape of compacta, Fund. Math. 67 (1970), 265-278. MR 42 \# 1077.

10. - On the concept of shape for metrizable spaces, Bull. Acad. Polon. Sci. Sér. Sci. Math. Astronom. Phys. 18 (1970), 127-132. MR 42 \#443.

11. , On the n-movability, Bull. Acad. Polon. Sci. Sér. Sci. Math. Astronom. Phys. 20 (1972), 859-864. MR 47 \#2540.

12. __ On positions of sets in spaces, Fund. Math. 79 (1973), 141-158. MR 48 \# 1154.

13. On fundamental deformation retracts and on some related notions, Fund. Math. 86 (1974), 261-270.

14. - On several problems in the theory of shape, Studies in Topology (Proc. Conf., Univ. North Carolina, 1974), Academic Press, 1975, pp. 67-79.

15. - Theory of shape, Math. Monographs, vol. 59, PWN, Warsaw, 1975.

16. T. A. Chapman, On some applications of infinite-dimensional manifolds to the theory of shape, Fund. Math. 76 (1972), 181-183. MR 47 \#9530.

17. _ Shapes of finite dimensional compacta, Fund. Math. 76 (1972), 261-276. MR 47 \#9531.

18. Some results on shapes and fundamental absolute retracts, Bull. Acad. Polon. Sci. Sér. Sci. Math. Astronom. Phys. 20 (1972), 37-40. MR 46 \# 8170.

19. H. Cook, G. Feuerbacher and W. Kuperberg, Shapes of finite dimensional continua have shape stable representatives, Bull. Acad. Polon. Sci. Sér. Sci. Math. Astronom. Phys. (to appear).

20. D. Coram, R. J. Daverman and P. F. Duvall, Jr., A loop condition for embedded compacta, Proc. Amer. Math. Soc. 53 (1975), 205-212.

21. C. Cox, Three questions of Borsuk concerning movability and fundamental retraction, Fund. Math. 80 (1973), 169-179. MR 48 \#9646.

22. D. A. Edwards and R. Geoghegan, Shapes of complexes, ends of manifolds, homotopy limits and the Wall obstruction, Ann. of Math. (2) 101 (1975), 521-535.

23. R. H. Fox, On shape, Fund. Math. 74 (1972), 47-71; errata, ibid. 75 (1972), p. 85 . MR 45 \# 5973; 46 \#6282.

24. R. H. Fox, Shape theory and covering spaces, Topology Conf. (Virginia Polytech. Inst. and State Univ., Blacksburg, Va., 1973), Lecture Notes in Math., vol. 375, Springer, Berlin, 1974, pp. 71-90. MR 51 \#4249.

25. R. Geoghegan and R. R. Summerhill, Concerning the shapes of finite-dimensional compacta, Trans. Amer. Math. Soc. 179 (1973), 281-292. MR 48 \#2987. 
26. G. R. Gordh, Jr. and S. Mardešic, On the shape of movable Hausdorff curves, Bull. Acad. Polon. Sci. Sér. Sci. Math. Astronom. Phys. 23 (1975), 169-176.

27. J. G. Hollingsworth and T. B. Rushing, Embeddings of shape classes of compacta in the trivial range, Preprint, Notices Amer. Math. Soc. Aug. 1975, p. A-526. Abstract \#75T-G69.

28. Y. Kodama, On $\Delta$-spaces and fundamental dimension in the sense of Borsuk, Fund. Math. 89 (1975), 13-22.

29. Y. Kodama and T. Watanabe, A note on Borsuk's $n$-movability, Bull. Acad. Polon. Sci. Sér. Sci. Math. Astronom. Phys. 22 (1974), 289-294. MR 49 \# 11462.

30. G. Kozlowski and J. Segal, n-movable compacta and ANR-systems, Fund. Math. 85 (1974), 235-243. MR 50 \# 11137.

31. J. Krasinkiewicz, Curves which are continuous images of tree-like continua are movable, Fund. Math. 89 (1975), 234-260.

32. V.-T. Liem, Certain continua in $S^{n}$ of the same shape have homeomorphic complements (preprint).

33. S. Mardešić, Retracts in shape theory, Glasnik Mat. Ser. III 6 (26) (1971), 153-163. MR 45 \# 5974.

34. , n-dimensional LC $C^{n-1}$ compacta are movable, Bull. Acad. Polon. Sci. Sér. Sci. Math. Astronom. Phys. 19 (1971), 505-509. MR 46 \#869.

35. Strongly movable compacta and shape retracts, Proc. Internat. Sympos. on Topology and its Applications (Budva, 1972), Savez. Društava Mat. Fiz. i Astronom., Belgrade, 1973, pp. 163-166. MR 48 \# 12462.

36. Shapes for topological spaces, General Topology and Appl. 3 (1973), 265-282. MR 48 \#2988.

37. _ Equivalence of two notions of shape for metric spaces, Bull. Acad. Polon. Sci. Sér. Sci. Math. Astronom. Phys. 21 (1973), 1137-1142.

38. S. Mardešić and J. Segal, Shapes of compacta and ANR-systems, Fund. Math. 72 (1971), no. 1, 41-59. MR 45 \#7686.

39. __ Equivalence of the Borsuk and the ANR-system approach to shapes, Fund. Math. 72 (1971), no. 1, 61-68. MR 46 \#850.

40. , Movable compacta and ANR-systems, Bull. Acad. Polon. Sci. Sér. Sci. Math. Astronom. Phys. 18 (1970), 649-654. MR 44 \# 1026.

41. D. R. McMillan, Jr., One-dimensional shape properties and three-manifolds, Studies in Topology (Proc. Conf., Univ. North Carolina, 1974), Academic Press, 1975, pp. 367-381. MR 51 \#6824.

42., A locally connected non-movable continuum that fails to separate $E^{3}$, Fund. Math. (to appear).

43. D. R. McMillan and N. Shrikhande, On the simple connectivity of a quotient space (preprint).

44. K. Morita, On shapes of topological spaces, Fund. Math. 86 (1974), 251-259.

45. S. Nowak, Some properties of fundamental dimension, Fund. Math. 85 (1974), 211-227.

46. , On the fundamental dimension of approximatively 1-connected compacta, Fund. Math. 89 (1975), 61-79.

47. , Remarks on the fundamental dimension of components of movable compacta, Bull. Acad. Polon. Sci. Sér. Sci. Math. Astronom. Phys. 23 (1975) (to appear).

48. T. B. Rushing, The compacta $X$ in $S^{n}$ for which $\operatorname{Sh}(X)=\operatorname{Sh}\left(S^{k}\right)$ is equivalent to $S^{n}-X \approx S^{n}-S^{k}$ (preprint).

49. N. Shrikhande, Homotopy properties of decomposition spaces, Notices Amer. Math. Soc., April 1975, p. A-392. Abstract \#75T-G38.

50. M. Strok, A remark on Borsuk's paper "On fundamental deformation retracts and on some related notions", Bull. Acad. Polon. Sci. Sér. Sci. Math. Astronom. Phys. 23 (1975), 767-769.

51. A. Trybulec, On shapes of movable curves, Bull. Acad. Polon. Sci. Sér. Sci. Math. Astronom. Phys. 21 (1973), 727-733.

52. G. A. Venema, Embeddings of compacta with shape dimensions in the trivial range (preprint).

53. __ Weak flatness for shape classes of sphere-like continua (preprint).

54. J. E. West, Compact ANR's have finite type, Bull. Amer. Math. Soc. 81 (1975), 163-165. MR 50 \# 11250.

55. J. Segal, Shape theory bibliography, Univ. of Washington, 1975 (mimeographed). 


\section{SUPPLEMENTARY BIBLIOGRAPHY}

56. B. J. Ball, Shapes of saturated subsets of compacta, Colloq. Math. 29 (1974), 241-246, 307. MR $49 \# 1470$.

57. _ , Proper shape retracts, Fund. Math. 89 (1975), 177-189.

58. R. Bennett, On the fundamental dimension of compact spaces, Bull. Acad. Polon. Sci. Sér.

Sci. Math. Astronom. Phys. 21 (1973), 65-68. MR 47 \#814.

59. R. Bennett and K. Borsuk, On homotopically compact spaces, Bull. Acad. Polon. Sci. Sér. Sci. Math. Astronom. Phys. 20 (1972), 865-869. MR 48 \# 12459.

60. S. A. Bogatyì, Approximative and fundamental retracts, Mat. Sb. (N.S.) 93 (135) (1974), 90-102, 152 = Math. USSR Sbornik 22 (1974), 91-103. MR 49 \#1468.

61. K. Borsuk, Problems concerning the notion of the shape of compacta, Proc. Internat. Sympos. on Topology and its Applications (Herceg-Novi, 1968), Savez Društaya Mat. Fiz. i Astronom., Belgrade, 1969, pp. 98-104. MR 43 \#1138.

62. _ On the shape of the suspension, Colloq. Math. 21 (1970), 247-252. MR 42 $\# 2483$.

63. , On the homotopy types of some decomposition spaces, Bull. Acad. Polon. Sci. Sér.

Sci. Math. Astronom. Phys. 18 (1970), 235-239. MR 42 \#2427.

64. $\quad$ A note on the shape of quasi-homeomorphic compacta, Comment. Math. Prace Mat. 14 (1970), 25-34. MR 44 \#986.

65. , Remark on a theorem of S. Mardesić, Bull. Acad. Polon. Sci. Sér. Sci. Math. Astronom. Phys. 19 (1971), 475-483. MR 48 \#2986.

66. - Theory of shape, Lecture Notes Series, no. 28, Matematisk. Institut, Aarhus Univ., Aarhus, 1971, 145 pp. MR 45 \#2679.

67. _ Some remarks concerning the theory of shape in arbitrary metrizable spaces, General topology and its relations to modern analysis and algebra, III (Proc. Third Prague Topological Sympos., 1971) Academia, Prague, 1972, pp. 77-81.

68. _ - On homotopy properties of compact subsets of the Hilbert space, Sympos. on Inf. Dim. Topology, Ann. of Math. Studies, no. 69, Princeton Univ. Press, Princeton, N.J., 1972, pp. 25-36.

69. - Remarks on the notion of position, Proc. Internat. Sympos. on Topology and its Applications (Budva, 1972), Savez Društava Mat. Fiz. i Astronom., Belgrade, 1973, pp. 40-44. MR 48 \#7188.

70. , On some hereditable shape properties, Ann. Polon. Math. 29 (1974), 83-86. MR 50 \#3184.

71. , Some remarks on shape properties of compacta, Fund. Math. 85 (1974), 185-195. MR 50 \#5730.

72. , Concerning the shapes of n-dimensional spheres, Fund. Math. 85 (1974), 197-202. MR 50 \#5731.

73. , On nearly extendable maps, Bull. Acad. Polon. Sci., Sér. Sci. Math. Astronom Phys. 23 (1975), 753-760.

74. - Some quantative properties of shapes, Fund. Math. (to appear).

75. - On a metrization of the hyperspace of a compactum, Fund. Math. (to appear).

76. K. Borsuk and W. Holsztyński, Concerning the ordering of shapes of compacta, Fund. Math. 68 (1970), 107-115. MR 42 \#2432.

77. T. A. Chapman, Characterizing shapes of compacta, Bull. Amer. Math. Soc. 78 (1972), 847-851. MR 45 \#9297.

78. - Shapes of some decomposition spaces, Bull. Acad. Sci. Polon. Sér. Sci. Math. Astronom. Phys. 20 (1972), 653-656. MR 47 \#2541.

79. J. Dydak, On the shape of decomposition spaces, Bull. Acad. Polon. Sci. Sér. Sci. Math. Astronom. Phys. 23 (1975), 293-298.

80. _ Movability and the shape of decomposition spaces, Bull. Acad. Polon. Sci., Sér.

Sci. Math. Astronom. Phys. 23 (1975), 441-452.

81. _ Some remarks on the shape of decomposition spaces, Bull. Acad. Polon. Sci., Sér. Sci. Math. Astronom. Phys. 23 (1975), 561-564.

82. J. Dydak and A. Trybulec, On regularly movable compacta, Bull. Acad. Polon. Sci. Sér. Sci. Math. Astronom Phys. 23 (1975), 907-909.

83. R. Geoghegan and R. C. Lacher, Compacta with the shape of finite complexes (preprint). 
84. A. Gmurczyk, On the shape of finite bouquets of $n$-spheres, Bull. Acad. Polon. Sci., Sér. Sci. Math. Astronom. Phys. 22 (1974), 1251-1254. MR 51 \#6716.

85. , Approximative retracts and fundamental retracts, Colloq. Math. 23 (1971), 61-63. MR 46 \# 6274.

86. , On bouquets, Fund. Math. (to appear).

87. S. Godlewski, On shapes of solenoids, Bull. Acad. Polon. Sci. Sér. Sci. Math. Astronom. Phys. 17 (1969), 623-627. MR 41 \#9252.

88. _ Solenoids of comparable shapes are homeomorphic, Bull. Acad. Polon. Sci., Sér. Sci. Math. Astronom. Phys. 18 (1970), 565-566. MR 43 \#6920.

89. , Mutational retracts and extensions of mutations, Fund. Math. 84 (1974), 47-65. MR 49 \#9800.

90. , On shape of MAR and MANR-spaces, Fund. Math. 88 (1975), 87-94.

91. An example resolving some Borsuk's problems concerning the shape of metrizable spaces, Bull. Acad. Polon. Sci. Sér. Sci. Math. Astronom. Phys. 23 (1975), 417-420.

92. , A characterization of MAR- and MANR-spaces by extendability of mutations, Fund. Math. 89 (1975), 229-232.

93. S. Godlewski and S. Nowak, On two notions of shape, Bull. Acad. Polon. Sci. Sér. Sci. Math. Astronom. Phys. 20 (1972), 387-393. MR 47 \# 1016.

94. D. Handel and J. Segal, An acyclic continuum with non-movable suspensions, Bull. Acad. Polon. Sci. Sér. Sci. Math. Astronom. Phys. 21 (1973), 171-172. MR 47 \# 5813.

95. D. W. Henderson, Applications of infinite-dimensional manifolds to quotient spaces of complete ANR's, Bull. Acad. Polon. Sci. Sér. Sci. Math. Astronom. Phys. 19 (1971), 748-753. MR 46 \#6263.

96. D. M. Hyman, On decreasing sequences of compact absolute retracts, Fund. Math. 64 (1969), 91-97. MR 40 \#6518.

97. _ A remark on Fox's paper on shape, Fund. Math. 75 (1972), no. 3, 205-208. MR 46 \#2635.

98. J. Keesling, A non-movable trivial-shape decomposition of the Hilbert cube, Bull. Acad. Polon. Sci. Sér. Sci. Math. Astronom. Phys. 23 (1975), 997-998.

99. Y. Kodama, On the shape of decomposition spaces, J. Math. Soc. Japan 26 (1974), 636-646. MR 50 \# 14732.

100. , On embeddings of spaces into ANR and shapes, J. Math. Soc. Japan 27 (1975), $533-544$.

101. , Decomposition spaces and shape in the sense of Fox (preprint).

102. J. Krasinkiewicz, Remark on the notion of fundamentally compact spaces, Bull. Acad. Polon. Sci., Sér. Sci. Math. Astronom. Phys. 21 (1973), 69-71. MR 47 \# 4217.

103. Shape properties of hyperspaces, Fund. Math. (to appear).

104. Continuous images of continua and 1-movability, Fund. Math. (to appear).

105. - On a construction of ANR-sets, Fund. Math. (to appear).

106. S. Lapinskis, On the shape of closed plane pseudo-continua, Bull. Acad. Polon. Sci. Sér. Sci. Math. Astronom. Phys. 22 (1974), 295-304. MR 49 \#11463.

107. S. Mardešic, $A$ survey of the shape theory of compacta, General Topology and its Relations to Modern Analysis and Algebra, III (Proc. Third Prague Topological Sympos, 1971), Academia, Prague, 1972, pp. 291-300. MR 51 \# 1726.

108. , On the shape of the quotient space $S^{n} / A$, Bull. Acad. Polon. Sci. Sér. Sci. Math. Astronom. Phys. 19 (1971), 623-629. MR 46 \# 8155.

109. _ A non-movable compactum with movable suspension, Bull. Acad. Polon. Sci. Sér. Sci. Math. Astronom. Phys. 19 (1971), 1101-1103. MR 46 \#6325.

110. , Decreasing sequences of cubes and compacta of trivial shape, General Topology and Appl. 2 (1972), 17-23. MR 46 \# 870.

111. M. Moszyńska, On shape and fundamental deformation retracts. I, Fund. Math. 75 (1972), 145-167. MR 50 \# 11138.

112. On shape and fundamental deformation retracts. II, Fund. Math. 77 (1973), 235-240. MR 48 \#9647.

113. J. Olędzki, On shape of the join of compacta, Bull. Acad. Polon. Sci. Sér. Sci. Math. Astronom. Phys. 20 (1972), 853-858. MR 48 \# 1156.

114. On the space of components of an $\Re$-movable compactum, Bull. Acad. Polon. Sci. Sér. Sci. Math. Astronom. Phys. 22 (1974), 1239-1244. MR 50 \# 14649. 
115. , On the movability and other similar shape properties, Fund. Math. 88 (1975),

179-191.

116. M. Orlowski, $n$ fundamental matching of compacta, Bull. Acad. Polon. Sci. Sér. Sci. Math. Astronom. Phys. 21 (1973), 255-262. MR 50 \# 1199.

117. R. H. Overton and J. Segal, A new construction of movable compacta, Glasnik. Mat. Ser. III 6 (26) (1971), 361-363. MR 48 \#1157.

118. H. Patkowska, $A$ homotopy extension theorem for fundamental sequences, Fund. Math. 64 (1969), 87-89. MR 39 \#3487.

119. R. B. Sher, Property $S U V^{\alpha}$ and proper shape theory, Trans. Amer. Math. Soc., 190 (1974), 345-356. MR 49 \#6140.

120. _ Proper shape theory and neighborhoods of sets in Q-manifolds, Bull. Acad. Polon. Sci. Sér. Sci. Math. Astronom. Phys. 23 (1975), 270-276.

121. _ Extensions, retracts, and absolute neighborhood retracts in proper shape theory (preprint).

122. S. Singh, On a question of Borsuk on FAR's, Bull. Acad. Polon. Sci. Sér, Sci. Math. Astronom. Phys. 23 (1975), 267-269.

123. S. Spiez, On a plane compactum with the maximal shape, Bull. Acad. Polon. Sci. Ser. Sci. Math. Astronom. Phys. 21 (1973), 615-620. MR 48 \# 12465.

125. , Movability and uniform movability, Bull. Acad. Polon. Sci. Sér. Sci. Math. Astronom. Phys. 22 (1974), 43-45. MR 49 \# 11466.

126. - On a characterization of shapes of several compacta, Bull. Acad. Polon. Sci. Sér. Sci. Math. Astronom. Phys. (to appear).

127. A. Trybulec, On the uniqueness of the decomposition of movable shapes into a product of 1-dimensional shapes, Bull. Acad. Polon. Sci. Sér. Sci. Math. Astronom. Phys. 23 (1975), 69-73.

Department of Mathematics, University of Georgia, Athens, Georgia 30602 